\title{
SARS-CoV-2 Omicron variant causes mild pathology in the upper and lower respiratory tract of Syrian golden hamsters (Mesocricetus auratus)
}

\section{Federico Armando}

University of Veterinary Medicine Hannover https://orcid.org/0000-0002-2578-4409

\section{Georg Beythien}

University of Veterinary Medicine Hannover

\section{Franziska Kaiser}

Research Center for Emerging Infections and Zoonoses, University of Veterinary Medicine, Hanover, Germany

\section{Lisa Allnoch}

Department of Pathology, University of Veterinary Medicine, Foundation, Hanover, Germany

\section{Laura Heydemann}

Department of Pathology, University of Veterinary Medicine, Foundation, Hanover, Germany

\section{Malgorzata Rosiak}

Department of Pathology, University of Veterinary Medicine, Foundation, Hanover, Germany

\section{Svenja Becker}

Department of Pathology, University of Veterinary Medicine, Foundation, Hanover, Germany

\section{Mariana Gonzalez-Hernandez}

Research Center for Emerging Infections and Zoonoses, University of Veterinary Medicine, Hanover, Germany

\section{Mart Lamers}

Erasmus University Medical Center https://orcid.org/0000-0002-1431-4022

\section{Bart Haagmans}

Erasmus University Medical Center https://orcid.org/0000-0001-6221-2015

\section{Kate Guilfoyle}

Viroclinics Xplore

\section{Geert van Amerongen}

ViroClinics DDL

\section{Malgorzata Ciurkiewicz}

Department of Pathology, University of Veterinary Medicine, Foundation, Hanover, Germany

Albert Osterhaus

University of Veterinary Medicine Hanover

Wolfgang Baumgärtner ( $\nabla$ wolfgang.Baumgaertner@tiho-hannover.de ) 


\section{Article}

\section{Keywords:}

Posted Date: February 1st, 2022

DOl: https://doi.org/10.21203/rs.3.rs-1315280/v1

License: (c) (1) This work is licensed under a Creative Commons Attribution 4.0 International License. Read Full License

Version of Record: A version of this preprint was published at Nature Communications on June 20th, 2022. See the published version at https://doi.org/10.1038/s41467-022-31200-y. 


\section{Abstract}

Since its discovery in 2019, multiple variants of severe acute respiratory syndrome coronavirus-2 (SARSCoV-2) have been identified worldwide. The present study investigates virus spread and associated pathology in the upper and lower respiratory tracts in the early phase of SARS-CoV-2 Omicron infection in the Syrian golden hamster (Mesocricetus auratus) in comparison to previous identified variants of concern (VOCs). Syrian golden hamsters were infected intranasally with SARS-CoV-2 614G or with VOCs Gamma, Delta and Omicron. Pathological changes both in the upper and lower respiratory tract of VOC Omicron infected hamsters were milder than those caused by the other investigated strains. VOC Omicron infection caused only a mild rhinitis with mild involvement of the olfactory epithelium and minimal lesions in the lung with frequent sparing of the alveolar compartment. Similarly, viral antigen detection as well as infectious SARS-CoV-2 titers were lower in upper and lower respiratory tract of VOC Omicron infected hamsters. These findings demonstrate that the SARS-CoV-2 VOC Omicron variant has a decreased pathogenicity for both the upper and lower respiratory tract of Syrian golden hamsters.

\section{Introduction}

Since the first case of COVID-19 was reported more than 330 million SARS-CoV-2 infections and 5,5 million deaths have been detected worldwide ${ }^{1}$. After the initial identification of the SARS-CoV-2 strain coming from Wuhan, several variants of concern (VOCs) have been identified including Alpha (B.1.1.7) in the United Kingdom, Beta (B.1.351) in South Africa, Gamma (P.1) in Brazil, and Delta (B.1.617.2) in India. These were associated with decreased effectiveness of medical interventions, epidemiological changes and/or a variable clinical course, collectively necessitating adjustment of public health and societal measures ${ }^{2,3}$. Recently, a new SARS-CoV-2 variant was identified in Botswana and later in South Africa, that was designated by the World Health Organization (WHO) VOC Omicron (B.1.1.529) based on an observed, unusually high number of mutations, high transmissibility and partial escape from pre-existing immunity ${ }^{4-6}$. First reports about clinical and epidemiological features of the infection indicate a relatively mild disease course and increased human-to-human virus spread ${ }^{7}$. So far, the potentially reduced pathogenicity of the VOC Omicron has not been addressed thoroughly in vivo experimental studies ${ }^{8}$. The WHO has officially declared that the first clinical data of VOC Omicron cases indicate its association with relatively mild disease ${ }^{9,10}$, but these data have not yet been corroborated by in-depth pathology studies.

Moreover, information about the relative pathogenicity and epidemiological significance of VOC Omicron compared to previous VOCs, which should be a major guide to public health response, is also urgently needed. Besides the implementation of non-pharmaceutical intervention protocols, this includes the development of future vaccination and antiviral treatment strategies for the ongoing pandemic. Among the experimental animal models, the use of non-human primates or ferrets, generally offering effective models for pre-clinical evaluation of vaccines and therapeutics for respiratory infections, provide limited options for pathogenicity studies. These models mimic asymptomatic to mild COVID-19 clinical courses only and therefore should not be considered the first choice to assess VOC Omicron pathogenicity and 
test COVID-19 vaccines and therapeutics ${ }^{11-15}$. The Syrian golden hamster (Mesocricetus auratus) model on the other hand, has been shown to mimic moderate to severe COVID-19 ${ }^{16}$, for evaluation of COVID-19 vaccines and therapeutics ${ }^{17}$, and recently for assessment of the pathogenicity of SARS-CoV-2 variants, such as VOC Omicron ${ }^{18}$.

The present study aims to obtain crucial information upon the pathogenicity of VOC Omicron, focusing specifically on the respiratory tract. For this reason, the upper and the lower respiratory tract, of eight to ten weeks old Syrian golden hamsters were thoroughly investigated following intranasal inoculation with $10^{4} \mathrm{TCID}_{50}$ of either SARS-CoV-2 614G, or VOC Gamma (P.1, Brazil), VOC Delta (B.1.617.2, India) or VOC Omicron (B.1.1.529, South Africa) variants.

\section{Results}

1. VOC Omicron infection in hamsters does not cause weight loss and induces minimal macroscopic lesions in lungs

Upon daily recording, hamsters infected with SARS-CoV-2 614G, infection with VOCs Delta and Gamma showed a significant body weight loss that reached an average of $6,5 \% ; 11,2 \%$ and $10,8 \%$ after four days, respectively. In contrast, SARS-CoV-2 Omicron infected hamsters showed on an average increase in body weight of 3,1\% (Supplementary Table S1, Figure 1).

At necropsy, four days post infection (dpi) with SARS-CoV-2 614G and VOCs Gamma and Delta respectively, macroscopically the lungs of all hamsters showed moderate to severe, multifocal to coalescing areas of dark-red discoloration and consolidation with variable areas of pale discoloration on the surfaces. In contrast, lungs of hamsters infected with the VOC Omicron showed only minimal foci of reddish discoloration. No other organs displayed macroscopic changes.

These results indicate that acute VOC Omicron infection of hamsters leads to milder macroscopic lung lesions compared to infection with SARS-CoV-2 614G and VOCs Gamma and Delta, respectively.

\section{VOC Omicron infection in hamsters displays decreased pathogenicity for the upper respiratory tract}

In all SARS-CoV-2 infected hamsters, nasal turbinate lesions were observed. Microscopically, lesions were characterized by epithelial necrosis, neutrophilic/heterophilic exocytosis and sub-epithelial infiltration of macrophages and neutrophils/heterophils. The inflammation affected the respiratory and olfactory mucosa. Epithelial changes were more severe in the olfactory mucosa, which showed multifocal to coalescing areas of necrosis and sloughing, associated with intraluminal exudate composed of proteinaceous fluid, cell debris and degenerated neutrophils/heterophils. In the respiratory mucosa, only occasional cell death, small amounts of exudate and a reactive hyperplasia were observed. Rhinitis was moderate to severe in the majority of hamsters infected with either SARS-CoV-2 614G or VOCs Delta and Gamma. In contrast, hamsters infected with the VOC Omicron showed milder rhinitis. To confirm this, quantification of histopathological lesions was performed with a semi-quantitative scoring system. The 
overall nasal turbinate histopathological score of hamsters infected with VOC Omicron was significantly lower than that of hamsters infected with any other strain (Supplementary Table S2, Figure 2). Separate pathological evaluation of the respiratory and olfactory mucosa revealed that scores were significantly lower in both anatomical compartments in VOC Omicron infected hamsters compared to all other groups (Supplementary Table S2, Figure 2). Interestingly, SARS-CoV-2 614G infected animals showed significantly higher scores in the olfactory mucosa compared to VOCs Gamma and Delta (Supplementary Table S1).

In summary, VOC Omicron infected hamsters showed a significantly lower severity of lesions in the upper respiratory tract compared to those of the other investigated groups. This finding led to the expectation that also viral antigen expression and infectious virus titers in VOC Omicron infected animals would be lower.

Viral antigen was detected in the olfactory epithelium of the respective groups, while only scattered epithelial cells were immunolabelled in the respiratory mucosa. Quantification of the viral antigen in the respiratory epithelium of the nasal turbinates revealed no significant differences among the groups. However, quantification of viral antigen in the olfactory epithelium of VOC Omicron infected hamsters proved to be the lowest among the investigated groups, with statistically significant difference when compared to VOC Gamma and SARS-CoV-2 614G (Supplementary Table S2, Figure 3). In addition, SARSCoV-2 614G infected hamsters showed significantly higher numbers of SARS-CoV-2 NP ${ }^{+}$cells in the olfactory mucosa compared to VOC Gamma (Supplementary Table S2).

Infectious SARS-CoV-2 titers in the nasal turbinates confirmed the immunohistochemistry findings. VOC Omicron infected hamsters had the lowest infectious SARS-CoV-2 titers in the nasal turbinates with statistically significant differences when compared to all other groups (Supplementary Table S2, Figure 3). Interestingly, VOC Gamma infected hamsters showed significantly higher SARS-CoV-2 titers in the nasal turbinates than those infected with VOC Delta and SARS-CoV-2 614G (Supplementary Table S2).

Taken together, VOC Omicron infection displayed decreased pathogenicity for the upper respiratory tract of hamsters and was characterized by a mild rhinitis with reduced amount of viral antigen in the olfactory epithelium as well as decreased infectious SARS-CoV-2 titers. This lower pathogenicity of VOC omicron for the upper respiratory tract prompted us to study its pathogenicity for the lower respiratory tract.

\section{VOC Omicron infection in hamsters induces a moderate inflammation with intralesional viral antigen} expression in the trachea

Tracheal lesions were observed in all SARS-CoV-2 infected hamsters and were characterized by multifocal to coalescing sub-epithelial infiltration with macrophages, lymphocytes and neutrophils/heterophils with frequent neutrophilic/heterophilic exocytosis. In addition, scattered single cell death and ciliary loss were observed. The severity of tracheitis varied from mild to moderate in individual animals, regardless of the SARS-CoV-2 variant used. Quantification of tracheal histopathology by semiquantitative scoring showed no statistically significant differences among the groups 
(Supplementary Table S3, Figure 4). Nevertheless, it is important to note that the highest score was recorded in one animal belonging to the SARS-CoV-2 Omicron infected group (individual score $=12 \mathrm{VS}$ median score $=4$ ). Based on these findings we further analyzed viral antigen expression in VOC Omicron infected animals comparing it with that of VOCs Gamma and Delta and SARS-CoV-2 614G. Viral antigen was exclusively detected in VOC Omicron infected hamsters, resulting in a statistically significant difference compared to all other groups. However, the number of positive cells was below $5 \%$ of all epithelial cells in all animals of this group. (Supplementary Table S3, Figure 5).

Taken together, the trachea of VOC Omicron infected hamsters showed a mild to moderate tracheitis similar to the other groups, despite slightly higher numbers of SARS-CoV-2 NP ${ }^{+}$cells. After confirming the limited pathogenicity for the trachea, the investigations on the lower respiratory tract were completed with the lung investigations.

4. VOC Omicron infection of hamsters displays a lower pathogenicity for the lower respiratory tract than previous VOCs.

All SARS-CoV-2 infected hamsters showed inflammatory lesions in the lung, but the distribution and severity varied among the groups. Histopathological lesions were characterized by multifocal to coalescing broncho-interstitial pneumonia. Alveoli were often obscured by septal and luminal infiltrates of macrophages and neutrophils/heterophils admixed with extravasated erythrocytes and fibrin. Conductive airways showed occasional cell death and mucosal infiltration with neutrophils/heterophils and macrophages. Conductive airway epithelia frequently also showed piling up of cells and increased number of mitoses, interpreted as hyperplasia. In addition to alveoli and conductive airways, histopathological lesions were also observed in the vascular compartment. These were characterized by histiocytic-neutrophilic/heterophilic vasculitis with vascular wall degeneration, endothelialitis and luminal endothelial cell proliferation, interpreted as endothelial hypertrophy and hyperplasia. In addition, perivascular lympho-histiocytic cuffs, perivascular edema, and perivascular hemorrhages were also frequent.

Pneumonia was moderate to severe in the majority of hamsters infected with either SARS-CoV-2 614G or VOCs Delta and Gamma. Interestingly, a milder pneumonia was consistently observed in hamsters infected with VOC Omicron. The overall lung histopathological score of VOC Omicron infected hamsters was significantly lower compared to VOCs Gamma and Delta and SARS-CoV-2 614G infected hamsters (Supplementary Table S4, Figure 5). Separate evaluation of the lesions in alveoli, conductive airways and vessels showed that the scores were significantly lower in all three compartments of VOC Omicron infected animals compared to the other groups (Supplementary Table S4, Figure 5). Interestingly, lesions in VOC Omicron infected hamsters, seemed to be mainly centered on the airways and the vascular compartment, while alveoli showed no or minimal involvement (Supplementary Figure 1). In contrast, hamsters infected with either SARS-CoV-2 614G or VOCs Gamma and Delta showed equal involvement of all compartments. 
In summary, VOC Omicron infected hamsters showed a significantly decreased severity of lesions in the lower respiratory tract compared to the other investigated groups, with a notable sparing of alveoli. These findings led to the hypothesis that Omicron infects the lung to a lesser degree, as was also observed in the upper respiratory tract.

Viral antigen was observed in epithelial cells of the conductive airways and in type I and type II pneumocytes. The amount of SARS-CoV-2 NP antigen in the pulmonary conductive airways of VOC Omicron infected hamsters was the lowest among the investigated groups, with statistically significant difference when compared to VOC Gamma and SARS-CoV-2 614G (Supplementary Table S4, Figure 6). In addition, SARS-CoV-2 614G infected animals showed significantly higher numbers of SARS-CoV-2 NP ${ }^{+}$ compared to VOC Delta (Supplementary Table S4). The amount of viral antigen in the lung parenchyma, which included the alveolar and vascular compartment, was the lowest in VOC Omicron infected hamsters among all investigated groups, with a statistically significant difference only when compared to VOC Gamma infected ones (Supplementary Table S4, Figure 6).

Infectious SARS-CoV-2 titers in the lung confirmed immunohistochemistry findings. VOC Omicron infected hamsters showed the lowest infectious SARS-CoV-2 titers in the lung with statistically significant differences when compared to all other groups (Supplementary Table S4, Figure 6). Interestingly, VOC Delta infected hamsters showed significantly lower SARS-CoV-2 titers in the lung than VOC Gamma and SARS-CoV-2 614G infected hamsters (Supplementary Table S4).

Taken together, VOC Omicron infection in hamsters showed a decreased pathogenicity also for the lower respiratory tract and was characterized by a minimal to mild broncho-interstitial pneumonia with reduced SARS-CoV-2 NP antigen detection in the conductive airways and in the lung parenchyma, as well as decreased infectious SARS-CoV-2 titers. Collectively, these results demonstrate that VOC Omicron pathogenicity for the upper and lower respiratory tract is lower compared to SARS-CoV-2 614G and VOCs Gamma and Delta.

\section{VOC Omicron infection in hamster does not spread to extra-respiratory organs}

The histopathological analysis of extra-respiratory organs sampled during necropsy of VOC Omicron infected hamsters revealed no significant lesions attributable to SARS-CoV-2. Importantly, SARS-CoV-2 NP antigen was not detected in any tissue outside the respiratory tract.

Taken together, these results suggest that intranasal inoculation of hamsters with $10^{4} \mathrm{TCID}_{50}$ of SARSCoV-2 VOC Omicron variant neither causes histopathological lesions in, nor viral spread to, extrarespiratory organs.

\section{Discussion}

There are clear indications that SARS-CoV-2 VOC Omicron is less pathogenic in humans and leads to fewer hospital submissions than previous SARS-CoV-2 variants ${ }^{7}$, although limited information is available about the underlying mechanism. Therefore, animal models are used to compare the 
pathogenic potential of new emerging VOCs, such as VOC Omicron, with other previous variants. The pathogenicity of VOC Omicron has not fully been evaluated in the Syrian golden hamster model yet. In a previous study, infection with $10^{3} \mathrm{TCID}_{50}$ showed a lack of replication in the lung ${ }^{19}$. In our study, infection with $10^{4} \mathrm{TCID}_{50}$ did not cause weight loss in hamsters and showed a decreased pathogenicity for the upper and lower respiratory tract compared to VOCs Gamma and Delta and the SARS-CoV-2 614G. In addition, viral antigen detection and infectious SARS-CoV-2 titers were also lower in the upper and lower respiratory tract of VOC Omicron infected animals.

Body weight loss is a well-established parameter to assess clinical severity of SARS-CoV-2 infection in the hamster model during the acute phase of the infection ${ }^{17,20}$. The VOC Omicron infected hamsters of the present study show a mild weight increase of $3,1 \%$, while in the other groups weight loss observed ranging from $6,5 \%$ for SARS-CoV-2 $614 \mathrm{G}$ to $11,8 \%$ and $10,8 \%$ for the VOCs Delta and Gamma, respectively. These results are in agreement with the reported body weight loss of $\sim 10$ to $15 \%$ in hamsters infected with a variety of SARS-CoV-2 variants ${ }^{18}$. Moreover, the same study reported no weight loss in hamsters infected with VOC Omicron ${ }^{18}$. Taken together, the results of the current study indicated a less severe clinical course of the disease in VOC Omicron infected hamsters compared to other SARS-CoV-2 strains.

While the replication of VOC Omicron and associated histopathology in the hamster lung have been demonstrated in a recent publication ${ }^{18}$, histopathological changes and viral antigen distribution in the nasal turbinates have not been investigated. With other strains and variants, SARS-CoV-2 infected hamsters are reported to have a moderate to severe rhinitis at $4 \mathrm{dpi}$ with histopathological lesions and viral antigen detection mainly centered on the olfactory mucosa. SARS-CoV-2 primarily targets the sustentacular cells in the olfactory mucosa, leading to a transient, massive necrosis and disintegration of the epithelium ${ }^{21}$. This phenomenon in hamsters is associated with the development of anosmia, a frequent clinical feature of COVID-19 patients ${ }^{21-23}$. In addition, it is also reported in some patients and in hamsters that the development of prolonged loss of smell could be caused by virus persistence in the olfactory mucosa ${ }^{24}$. In our study, infection with SARS-CoV-2 $614 \mathrm{G}$ and VOCs Gamma and Delta also caused a widespread infection and damage in the olfactory epithelium, which is in agreement with previous reports. Interestingly, VOC Omicron infection in hamsters caused only a mild rhinitis with lesions mainly centered on the respiratory mucosa and a largely intact olfactory epithelium. The significantly reduced epithelial damage was also associated with decreased intraluminal exudates in the nasal turbinates. In addition, SARS-CoV-2 NP antigen detection was significantly decreased in the olfactory mucosa of VOC Omicron infected hamsters. These findings, together with lower titers of infectious SARSCoV-2 isolated from the nasal turbinates of VOC Omicron infected hamsters, indicated a decreased pathogenicity of VOC Omicron for the upper respiratory tract. Lower infectious SARS-CoV-2 titers isolated from the nasal turbinates of VOC Omicron infected hamsters were reported also by other studies ${ }^{18}$. Altogether, these results are tempting to speculate that VOC Omicron infection in hamsters results in a decreased viral shedding from the upper respiratory tract secretions. However, transmission experiments are required to further substantiate this hypothesis. Moreover, the decreased damage of the olfactory 
mucosa caused by VOC Omicron infection, most likely lowers the risk of developing anosmia. Preliminary data from human patients indeed suggests that anosmia appears to be a less frequent manifestation of VOC Omicron infection than reported for other strains and variants ${ }^{25-27}$. Given that anosmia is one of the most reported symptoms after SARS-CoV-2 infection ${ }^{28}$, a decrease of anosmia incidence might favor more asymptomatic and undetected courses of the disease.

Interestingly, like for the nasal turbinates, histopathological changes and viral antigen distribution in the trachea of VOC Omicron infected hamsters have not been investigated yet. However, the lack of significant differences in the pathological changes among the groups and the very low numbers of SARSCoV-2 NP ${ }^{+}$cells detected at 4 dpi might suggest that the trachea is most likely not one of the main target of this new SARS-CoV-2 VOC. Nevertheless, ciliary loss in the hamster tracheal epithelium following SARS-CoV-2 infection has been extensively discussed ${ }^{29}$, but whether VOC Omicron causes more severe or milder ciliary loss is still unknown. Future studies aiming at quantifying ciliary loss in VOC Omicron infected hamsters are warranted to shed some light on this unanswered question.

In contrast to nasal turbinates and trachea, lung histopathology in SARS-CoV-2 infected hamsters has been extensively described ${ }^{23,30-33}$. In addition, recent preliminary data on VOC Omicron infected hamsters also provided insights about lung lesions severity ${ }^{18,34}$. However, the reports lacked a detailed quantification of the lesion severity based on a thorough scoring system of distinct pulmonary anatomical compartments. The data of the current study are in agreement with the aforementioned studies and further corroborate the fact the VOC Omicron has a decreased pathogenicity for the lower respiratory tract. Interestingly, our data show that VOC Omicron-induced lung histopathological lesions were overall of lesser severity compared to those in animals infected with SARS-CoV-2 614G and the VOCs Gamma and Delta, as recently described in a comparative study among SARS-CoV-2 variants, including VOC Omicron, in hamsters and mice ${ }^{18}$. In particular, lung lesions were more centered in the conductive airways and vascular compartments, while there was a notable sparing of the alveoli. VOC Omicron-infected animals of our study showed either a complete absence or only minimal lesions in the alveoli. This limited alveolar damage appears to parallel the better clinical outcome in human patients, with lower risk of hospitalization and fatal outcomes. Nevertheless, the occurrence of long-COVID after VOC Omicron infection is still under debate ${ }^{35}$. Therefore, future long-term studies are warranted to confirm whether this decreased pathogenicity in the alveolar compartment may results in a decreased occurrence and milder manifestations of long-term sequelae post-infection.

SARS-CoV-2 viral antigen detection in the lung of infected hamsters is reported to be present in the lung conductive airways and alveolar cells until around $5 \mathrm{dpi}^{23}$, with a complete viral clearance around $7 \mathrm{dpi}^{23}$. The current study reported variable amount of SARS-CoV-2 NP antigen in the lung conductive airways and alveolar cells at $4 \mathrm{dpi}$ in all the investigated strains. VOC Omicron infected animals also showed a lower number of immunolabelled cells in all pulmonary compartments and lower infectious SARS-CoV-2 titers compared to those of the other groups. These results are in agreement with what has been recently published in a comparative study among SARS-CoV-2 variants, including VOC Omicron, in hamsters and 
mice $^{18}$. Altogether, these results show a decreased pathogenicity of VOC Omicron for the lower respiratory tract of hamsters.

SARS-CoV-2 infection of the CNS has been demonstrated in vitro in human brain organoids and in vivo in murine models and COVID-19 human patients ${ }^{36}$. Neuroinvasion through the olfactory route has also been demonstrated in hamsters, which was associated with neuroinflammation in the olfactory bulb ${ }^{24}$. In the current study, neither viral antigen nor associated neuropathology was detected in VOC Omicron-infected hamsters, which is most likely associated with the relatively limited infection observed in the olfactory mucosa.

SARS-CoV-2 has also been reported to spread to extra-respiratory organs such as heart and kidney in hamsters ${ }^{37}$ or testes ${ }^{38}$. The pathological investigations of extra-respiratory organs of hamsters infected with $10^{4} \mathrm{TCID}_{50}$ of VOC Omicron revealed neither significant lesions attributable to SARS-CoV-2 infection, nor detectable viral antigen in the investigated organs. However, the present study focused on the early phase of infection with VOC Omicron. Therefore, further studies including those with later time points and different virus dosages will be needed for a better understanding of the pathogenicity of this SARS-CoV-2 strain in the Syrian golden hamster.

In conclusion, SARS-CoV-2 VOC Omicron showed a lower pathogenicity for the upper and lower respiratory tract of Syrian golden hamsters without evidence for spread to other extra-respiratory organs.

\section{Material And Methods}

\section{Hamster study}

Approval for the experiment including SARS-CoV-2 614G Gamma and Delta variants was given by the Dutch authorities (Project license/working protocol (WP) number: 27700202114492-WP12). The Omicron study was conducted in Hannover at the University of Veterinary Medicine, Foundation with the approval of the Niedersächsisches Landesamt für Verbraucherschutz und Lebensmittelsicherheit (LAVES file number 21/3755). The animals were under veterinary observation during the experiment and all efforts were made to minimize distress. Eight to ten weeks old male Syrian golden hamsters were kept for ten days under BSL-3 conditions prior to the experiment for acclimatization. Hamsters $(n=22)$ were divided in 4 groups of 5-6 hamsters each. They were infected by administrating a suspension containing $10^{4}$ $\operatorname{TCID}_{50}$ with either, Gamma $(n=5)$, Delta $(n=5)$, Omicron $(n=6)$ VOCs or SARS-CoV-2 614G $(n=6)$ respectively. For four days the animals were monitored and weighed twice daily until euthanized at day 4 post infection using an overdose of Ketamine and Medetomidin followed by exsanguination. Immediately after death, necropsies were performed, the right lung lobe and nasal swabs were collected and frozen at $-70^{\circ} \mathrm{C}$ for virological analyses. Subsequently the left lung lobe, nasal turbinates, and tracheas were fixed in $10 \%$ buffered formalin (Chemie Vertrieb GmbH \& Co Hannover KG, Hannover, Germany). In addition, brain, liver, spleen, kidney, adrenal gland, stomach, small and large intestine, pancreas and testicle from hamsters infected with the VOC Omicron were also collected. Lungs were pre-fixed by injections of 10\% 
buffered formalin as recommended by Meyerholz and colleagues ${ }^{39}$ to ensure an optimal histopathological evaluation. Nasal samples, following formalin fixation, were decalcified for about 14 days prior routine tissue processing.

\section{SARS-CoV-2 variants}

SARS-CoV-2 614G (isolate Bavpat-1; European Virus Archive Global \#026 V-03883) was grown to passage 3 on VeroE6 cells, and VOCs were grown to passage 3 on Calu-3 cells. For stock production, infections were performed at a multiplicity of infection (moi) of 0.01 and virus was collected at 72 hours post-infection, clarified by centrifugation and stored at $-80^{\circ} \mathrm{C}$ in aliquots. Stock titers were determined as described below. All work with infectious SARS-CoV-2 was performed in a Class II Biosafety Cabinet under BSL-3 conditions at Viroclinics Xplore.

Viral genome sequences were determined using Illumina deep-sequencing as described before ${ }^{40}$. The $614 \mathrm{G}$ virus contained a spike $\mathrm{S} 686 \mathrm{G}$ change in $48 \%$ of reads compared with the passage 1 (kindly provided by Dr. Christian Drosten) and no other variants $>40 \%$. The VOC Gamma, Delta and Omicron variant passage 3 sequences were identical to the original respiratory specimens and no minor variants $>40 \%$ were detected. For VOC Omicron, the S1 region of spike was not covered well, due to primer mismatches. Therefore, the $\mathrm{S} 1$ region of the original respiratory specimen and passage 3 virus were confirmed to be identical by Sanger sequencing. VOC Gamma contained the following spike changes: L18F, T20N, P26S, D138Y, R190S, K417T, E484K, N501Y, D614G, H655Y, T1027I, V1176F. VOC Delta contained the following spike changes: T19R, G142D, del156-157, R158G, A222V, L452R, T478K, D614G, P681R and D950N. VOC Omicron contained the following spike mutations: A67VS, del69-70, T95I, G142-, del143-144, Y145D, del211, L212I, ins215EPE, G339D, S371L, S373P, S375F, K417N, N440K, G446S, S477N, T478K, E484A, Q493R, G496S, Q498R, N501Y, Y505H, T547K, D614G, H655Y, N679K, P681H, N764K, D796Y, N856K, Q954H, N969K, L981F. The VOCs gamma, delta and omicron sequences are available on Genbank under accession numbers OM442897, OM287123, and OM287553, respectively

\section{Virus infectivity titration}

Virus infectious titers were determined in Vero cells for the SARS-CoV-2 614G, VOCs Gamma and Delta variants. Due to the limited replication of VOC Omicron in Vero cells, this variant was propagated and titrated in Calu-3 cells. Cells were seeded in 96 well plate and incubated at $37^{\circ} \mathrm{C}$. Then $24 \mathrm{~h}$ after seeding, cell culture media was replaced by DMEM $+2 \%$ FBS in the case of Vero cells that were infected with 10 fold serial dilutions of lung or nasal turbinate homogenate tissue samples. Plates were further incubated in a humidified atmosphere at $37^{\circ} \mathrm{C}, 5 \% \mathrm{CO}$. Five days after infection, cytopathic effect was evaluated In the case of VOC Omicron titration, culture media was replaced for MEM $+2 \% \mathrm{FBS}$, $5 \mathrm{dpi}$ cells were fixed and stained using anti-SARS-CoV-2 Nucleocapsid antibody (Sinobiological). Viral titers (TCID50/ml) were calculated using the "Spearman-Kärber method" 41.

\section{Histopathology}


Formalin-fixed paraffın embedded samples were cut into $2 \mu \mathrm{m}$ thick serial sections and stained with hematoxylin and eosin (H\&E). Sections of the nasal turbinates, trachea, and lung were scanned using an Olympus VS200 Digital slide scanner (Olympus Deutschland GmbH, Hamburg, Germany) and evaluated in a blinded manner with a semi-quantitative scoring system with special emphasis on inflammation, degeneration and regeneration as previously described, with minor modification ${ }^{17}$. Histopathological semi-quantitative evaluations were performed by veterinary pathologists ( $G B, F A, M C, L A)$ and subsequently confirmed by a European board certified veterinary pathologist (WB). Nasal turbinates were evaluated on a full length longitudinal section of the nose including respiratory and olfactory epithelium. Trachea was evaluated on cross- and longitudinal sections along the entire length of the organ. Finally, the lung was evaluated on one cross section (at the level of the entry of the main bronchus) and one longitudinal section (along the main bronchus) of the entire left lung lobe. The applied scoring systems are provided in details in Supplementary table 5.

\section{Immunohistochemistry}

Immunohistochemistry of SARS-CoV-2 NP was performed using the Dako EnVision+ polymer system (Dako Agilent Pathology Solutions) and 3,3'-Diaminobenzidine tetrahydrochloride (Sigma-Aldrich, St. Louis, MO, United States) as previously described ${ }^{32,33}$. Monoclonal mouse primary antibody against SARS-CoV-2 NP (Sino Biological, Peking, China-40143-MM05; dilution $1: 16000$ ) was applied overnight at $4 \circ \mathrm{C}$.

\section{Digital image analysis}

For the quantification of immunolabelled cells in nasal turbinates as well as in tracheal and pulmonary tissue, slides were digitized using the Olympus VS200 (Olympus Deutschland GmbH, Hamburg, Germany) slide scanner. Image analysis was performed using the open source software package QuPath for digital pathology image analysis ${ }^{42}$. For all animals nasal turbinates and tracheal whole slides images as well as one longitudinal section (along the main bronchus) of the entire left lung lobe were evaluated. In brief, regions of interest (ROI), in the nasal turbinates (respiratory and olfactory mucosa) and the trachea (tracheal epithelum and subepithelial layer) were indicated by a veterinary pathologist. In the lung total tissue was detected automatically through digital thresholding and additional ROIs for conductive airways, including bronchi, bronchioles and terminal bronchioles were subsequently indicated by a veterinary pathologist. Lung parenchyma (alveolar and vascular compartments) was then obtained by subtraction of conductive airways from total lung tissue. The total numbers of immunolabelled and nonlabelled cells was determined by automated cell detection in all ROls, based on marker and tissue specific thresholding.

\section{Statistical Analyses}

Statistical analyses and graph design were performed using GraphPad Prism (GraphPad Software, San Diego, CA, USA) for Windows ${ }^{\text {TM }}$. Data was tested for significant differences using Kruskal-Wallis tests. 
Pairwise comparisons among groups were obtained by two-tailed Mann-Whitney-U tests. Statistical significance was accepted at exact p-values of $\leq 0.05\left(^{(*)}, \leq 0.01\left(^{(*)}\right.\right.$ and $\leq 0.001\left(^{(\star \star}\right)$, respectively.

\section{Declarations}

\section{Acknowledgements}

We thank Margarethe Jentzsch, Kristin Laudeley, Caroline Schütz, Julia Baskas, Kerstin Rohn, Kerstin Schöne, Jana Svea Harre for excellent technical support. This publication was supported by Deutsche Forschungsgemeinschaft and the University of Veterinary Medicine, Hannover, the foundation within the funding program of Open Access Publishing.

\section{Funding:}

This research was funded by the Deutsche Forschungsgemeinschaft (DFG; German Research Foundation) -398066876/GRK 2485/1; BMBF (Federal Ministry of Education and Research) project entitled RAPID (Risk assessment in re-pandemic respiratory infectious diseases), 01KI1723G, Ministry of Science and Culture of Lower Saxony in Germany (14 - 76103-184 CORONA-15/20) and the EU SC1-PHECORONAVIRUS-2020 MANCO, no 101003651

\section{Authors contributions:}

Conceptualization: WB, FA, GB, MC, ADMEO

Methodology: FA, GB, FK, MGH, ADMEO, BH, ML

Animal experiments: FK, MGH, ADMEO, KG, GvA

Investigation: WB, MC, FA, GB, LA, LH, MR, SB, FK, MGH, ADMEO

Visualization: FA, GB, LA, MC

Funding acquisition: WB, ADMEO

Project administration: WB, KG, FK, MGH

Supervision: WB, MC, ADMEO

Writing - original draft: FA, GB, LA, WB, MC, FK, MGH, ADMEO

Writing - review \& editing: WB, MC, FA, GB LA, LH, MR, SB, FK, MGH, ADMEO, BH, ML

\section{References}

1. (WHO), W. H. O. Coronavirus disease (COVID-19) pandemic., $<$ https://www.who.int/emergencies/diseases/novel-coronavirus-2019> (2022). 
2. Dao, T. L. et al. SARS-CoV-2 Infectivity and Severity of COVID-19 According to SARS-CoV-2 Variants: Current Evidence. J Clin Med 10, doi:10.3390/jcm10122635 (2021).

3. Raman, R., Patel, K. J. \& Ranjan, K. COVID-19: Unmasking Emerging SARS-CoV-2 Variants, Vaccines and Therapeutic Strategies. Biomolecules 11, doi:10.3390/biom11070993 (2021).

4. Kupferschmidt, K. Where did 'weird' Omicron come from? Science (New York, N.Y.) 374, 1179, doi:10.1126/science.acx9738 (2021).

5. (WHO), w. h. o. Update on SARS-COV-2 and Omicron VOC <https://www.who.int/publications/m/item/update-on-sars-cov-2-and-omicron-voc > (

6. Control, E. C. f. D. P. a. Implications of the emergence and spread of the SARS-CoV-2 B.1.1. 529 variant of concern (Omicron), for the EU/EEA, <.https://www.ecdc.europa.eu/en/publicationsdata/threat-assessment-brief-emergence-sars-cov-2-variant-b.1.1.529 > (

7. Abdullah, F. et al. Decreased severity of disease during the first global omicron variant covid-19 outbreak in a large hospital in tshwane, south africa. Int J Infect Dis 116, 38-42, doi:10.1016/j.jijid.2021.12.357 (2021).

8. Karim, S. S. A. \& Karim, Q. A. Omicron SARS-CoV-2 variant: a new chapter in the COVID-19 pandemic. Lancet (London, England) 398, 2126-2128, doi:10.1016/s0140-6736(21)02758-6 (2021).

9. (WHO), W. H. O. Classification of omicron (B.1.1.529): SARS-CoV-2 variant of concern. 2021, $<$ https://www.who.int/news/item/26-11-2021- classification-of-omicron-(b.1.1.529)-sars-cov-2variant-of-concern> $(2022)$.

10. Poudel, S. et al. Highly mutated SARS-CoV-2 Omicron variant sparks significant concern among global experts - What is known so far? Travel Med Infect Dis 45, 102234, doi:10.1016/j.tmaid.2021.102234 (2022).

11. Bewley, K. R. et al. Immunological and pathological outcomes of SARS-CoV-2 challenge following formalin-inactivated vaccine in ferrets and rhesus macaques. Sci Adv 7, eabg7996, doi:10.1126/sciadv.abg7996 (2021).

12. Everett, H. E. et al. Intranasal Infection of Ferrets with SARS-CoV-2 as a Model for Asymptomatic Human Infection. Viruses 13, doi:10.3390/v13010113 (2021).

13. Ciurkiewicz, M. et al. Ferrets are valuable models for SARS-CoV-2 research. Vet Pathol, 3009858211071012, doi:10.1177/03009858211071012 (2022).

14. Salguero, F. J. et al. Comparison of rhesus and cynomolgus macaques as an infection model for COVID-19. Nat Commun 12, 1260, doi:10.1038/s41467-021-21389-9 (2021).

15. Munster, V. J. et al. Subtle differences in the pathogenicity of SARS-CoV-2 variants of concern B.1.1.7 and B.1.351 in rhesus macaques. Sci Adv 7, eabj3627, doi:10.1126/sciadv.abj3627 (2021).

16. Muñoz-Fontela, C. et al. Animal models for COVID-19. Nature 586, 509-515, doi:10.1038/s41586020-2787-6 (2020).

17. Bošnjak, B. et al. Intranasal Delivery of MVA Vector Vaccine Induces Effective Pulmonary Immunity Against SARS-CoV-2 in Rodents. Front Immunol 12, 772240, doi:10.3389/fimmu.2021.772240 
(2021).

18. Halfmann, P. J. et al. SARS-CoV-2 Omicron virus causes attenuated disease in mice and hamsters. Nature, doi:10.1038/s41586-022-04441-6 (2022).

19. Abdelnabi, R. et al. The omicron (B.1.1.529) SARS-CoV-2 variant of concern does not readily infect Syrian hamsters. bioRxiv, 2021.2012.2024.474086, doi:10.1101/2021.12.24.474086 (2021).

20. Imai, M. et al. Syrian hamsters as a small animal model for SARS-CoV-2 infection and countermeasure development. Proc Natl Acad Sci U S A 117, 16587-16595, doi:10.1073/pnas.2009799117 (2020).

21. Bryche, B. et al. Massive transient damage of the olfactory epithelium associated with infection of sustentacular cells by SARS-CoV-2 in golden Syrian hamsters. Brain Behav Immun 89, 579-586, doi:10.1016/j.bbi.2020.06.032 (2020).

22. Reyna, R. A. et al. Recovery of anosmia in hamsters infected with SARS-CoV-2 is correlated with repair of the olfactory epithelium. Sci Rep 12, 628, doi:10.1038/s41598-021-04622-9 (2022).

23. Sia, S. F. et al. Pathogenesis and transmission of SARS-CoV-2 in golden hamsters. Nature $\mathbf{5 8 3}, \mathbf{8 3 4}$ 838, doi:10.1038/s41586-020-2342-5 (2020).

24. de Melo, G. D. et al. COVID-19-related anosmia is associated with viral persistence and inflammation in human olfactory epithelium and brain infection in hamsters. Sci Transl Med 13, doi:10.1126/scitranslmed.abf8396 (2021).

25. Agency, U. H. S. Investigation of SARS-CoV-2 variants: technical briefings. Technical briefing documents on novel SARS-CoV-2 variants., $<$ https://www.gov.uk/government/publications/investigation-of-sars-cov-2-variants-technicalbriefings> (2021).

26. MEDICINE, Y. Omicron: A Guide to What We Know Now, <https://www.yalemedicine.org/news/5things-to-know-omicron> (2022).

27. News, D. One major COVID symptom is missing from omicron variant, <https://www.deseret.com/2021/12/23/22849871/omicron-variant-symptoms-missing-covid-signsloss-taste-smell> (2021).

28. Lechien, J. R. et al. Severity of Anosmia as an Early Symptom of COVID-19 Infection May Predict Lasting Loss of Smell. Front Med (Lausanne) 7, 582802, doi:10.3389/fmed.2020.582802 (2020).

29. Robinot, R. et al. SARS-CoV-2 infection induces the dedifferentiation of multiciliated cells and impairs mucociliary clearance. Nat Commun 12, 4354, doi:10.1038/s41467-021-24521-x (2021).

30. Osterrieder, N. et al. Age-Dependent Progression of SARS-CoV-2 Infection in Syrian Hamsters. Viruses 12, doi:10.3390/v12070779 (2020).

31. Gruber, A. D., Firsching, T. C., Trimpert, J. \& Dietert, K. Hamster models of COVID-19 pneumonia reviewed: How human can they be? Vet Pathol, 3009858211057197, doi:10.1177/03009858211057197 (2021). 
32. Allnoch, L. et al. Vascular Inflammation Is Associated with Loss of Aquaporin 1 Expression on Endothelial Cells and Increased Fluid Leakage in SARS-CoV-2 Infected Golden Syrian Hamsters. Viruses 13, doi:10.3390/v13040639 (2021).

33. Becker, K. et al. Vasculitis and Neutrophil Extracellular Traps in Lungs of Golden Syrian Hamsters With SARS-CoV-2. Front Immunol 12, 640842, doi:10.3389/fimmu.2021.640842 (2021).

34. McMahan, K. et al. Reduced Pathogenicity of the SARS-CoV-2 Omicron Variant in Hamsters. bioRxiv, 2022.2001.2002.474743, doi:10.1101/2022.01.02.474743 (2022).

35. KAI KUPFERSCHMIDT, G. V. S., VOL 374, ISSUE 6573. 2021. How bad is Omicron? Some clues are emerging, and they're not encouraging, <https://www.science.org/content/article/how-bad-omicronsome-clues-are-emerging-and-they-re-not-encouraging $>$ (2021).

36. Song, E. et al. Neuroinvasion of SARS-CoV-2 in human and mouse brain. J Exp Med 218, doi:10.1084/jem.20202135 (2021).

37. Francis, M. E. et al. SARS-CoV-2 infection in the Syrian hamster model causes inflammation as well as type I interferon dysregulation in both respiratory and non-respiratory tissues including the heart and kidney. PLoS Pathog 17, e1009705, doi:10.1371/journal.ppat.1009705 (2021).

38. Campos, R. K. et al. SARS-CoV-2 Infects Hamster Testes. Microorganisms $\mathbf{9}$, doi:10.3390/microorganisms9061318 (2021).

39. Meyerholz, D. K., Sieren, J. C., Beck, A. P. \& Flaherty, H. A. Approaches to Evaluate Lung Inflammation in Translational Research. Vet Pathol 55, 42-52, doi:10.1177/0300985817726117 (2018).

40. GeurtsvanKessel, C. et al. Divergent SARS CoV-2 Omicron-specific T- and B-cell responses in COVID19 vaccine recipients. MedRxiv 2021.

41. Brandolini, M.et al. Correlating qRT-PCR, dPCR and Viral Titration for the Identification and Quantification of SARS-CoV-2: A New Approach for Infection Management. Viruses 13, doi:10.3390/v13061022 (2021).

42. Bankhead, P.et al. QuPath: Open source software for digital pathology image analysis. Sci Rep 7, 16878, doi:10.1038/s41598-017-17204-5 (2017).

\section{Figures}




\section{Body weight $(\%)$}

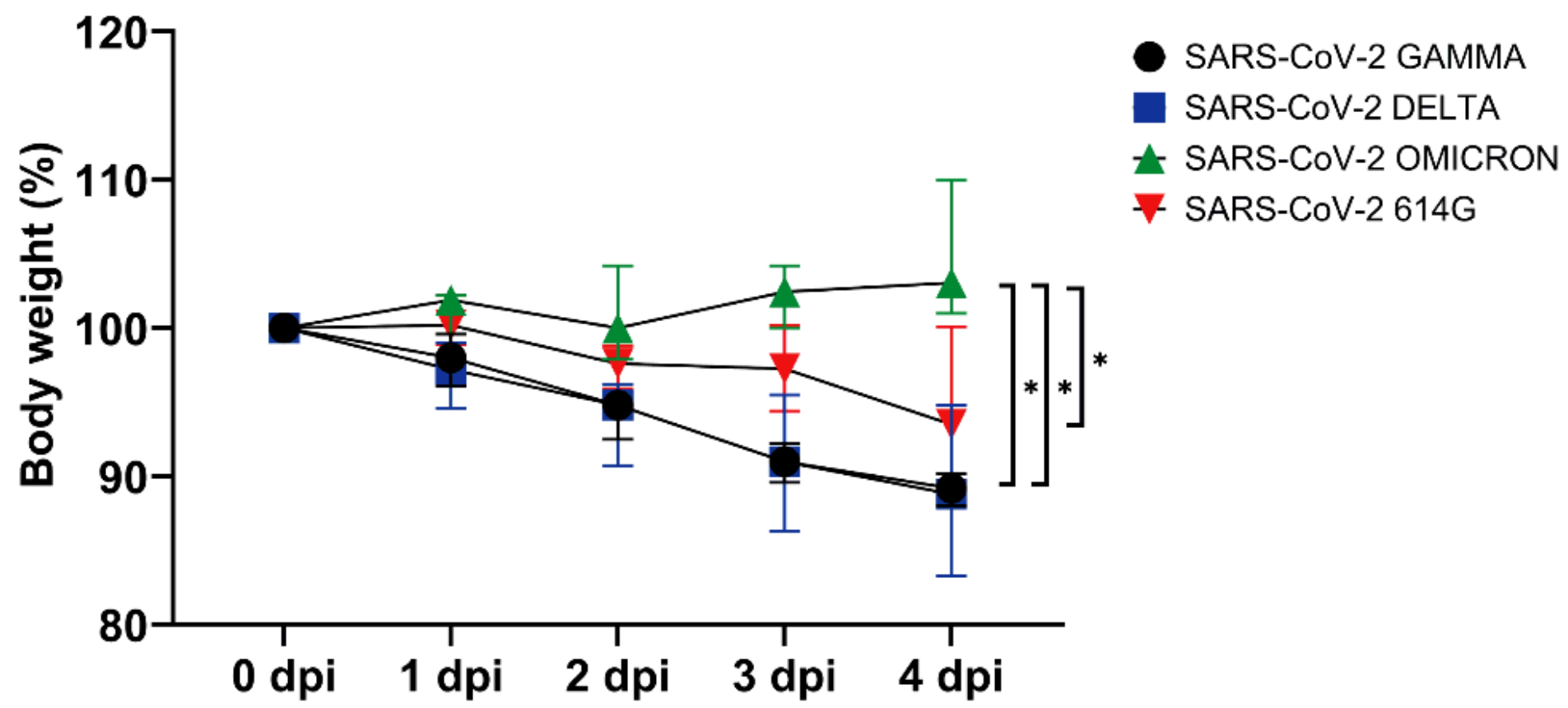

Figure 1

VOC Omicron infection in hamsters does not cause weight loss. Severe acute respiratory syndrome coronavirus-2 (SARS-CoV-2) variants of concern, Gamma and Delta infected hamsters showed significantly marked weight loss of $10,8 \%$ and $11,2 \%$, respectively. SARS-CoV-2 $614 \mathrm{G}$ infected hamsters revealed a significantly moderate weight loss with $6,5 \%$, whereas Omicron infected hamsters showed a mild increase in body weight with $3,1 \%$. Data are shown as median and range plots. Significant differences between the infection groups obtained by Mann-Whitney-U tests are indicated by * $\left({ }^{*} \mathrm{~S}\right.$ 0.05). 
A
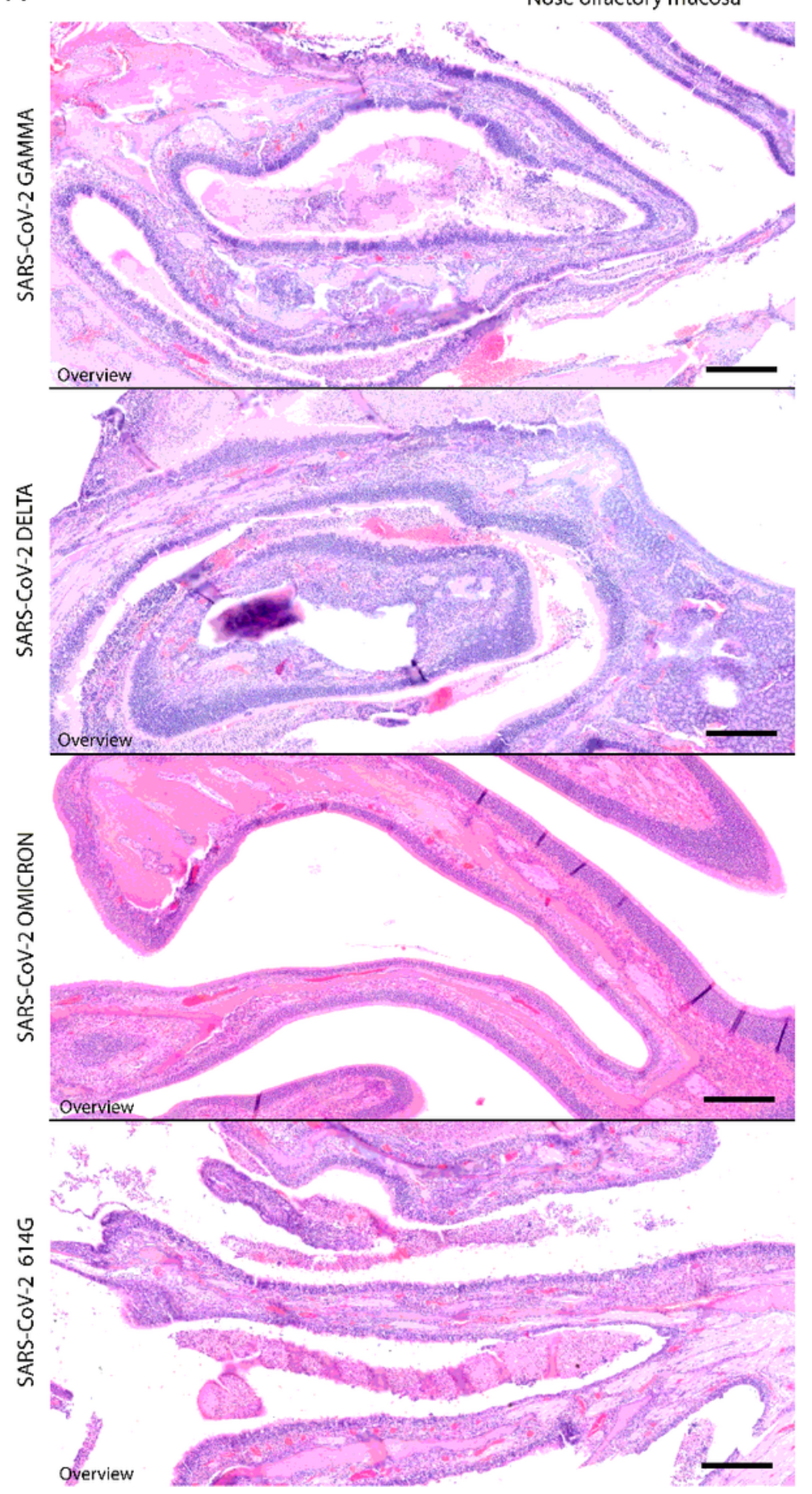

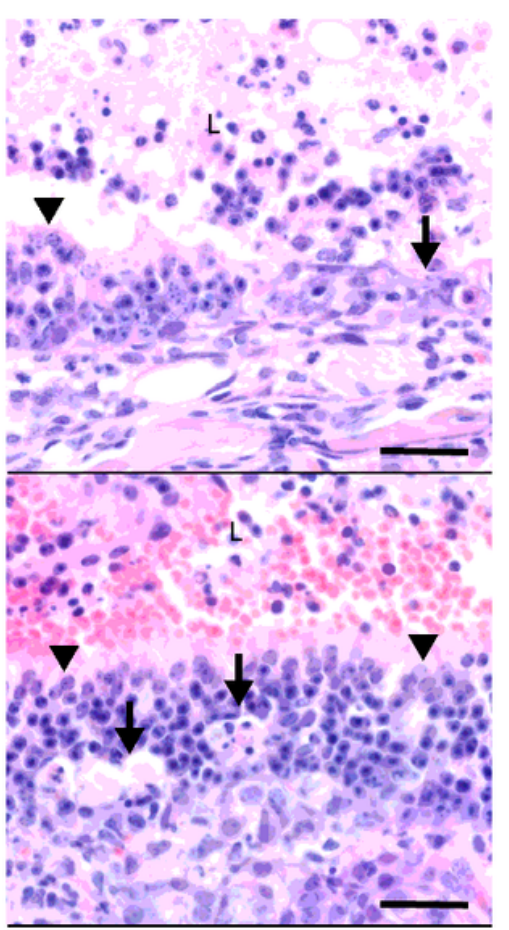

B
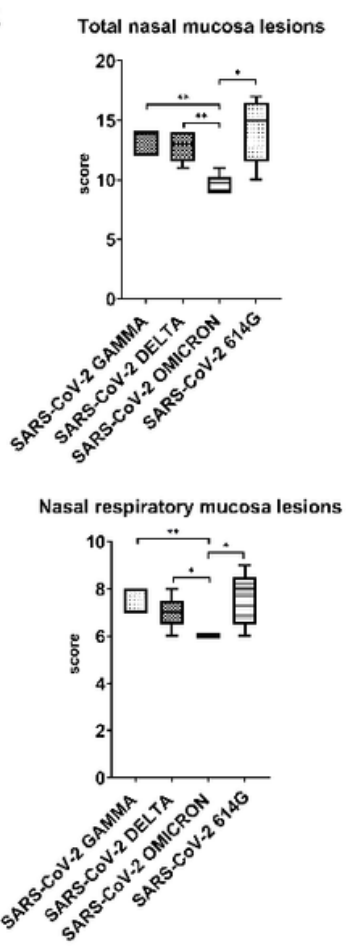

Nasal olfactory mucosa lesions
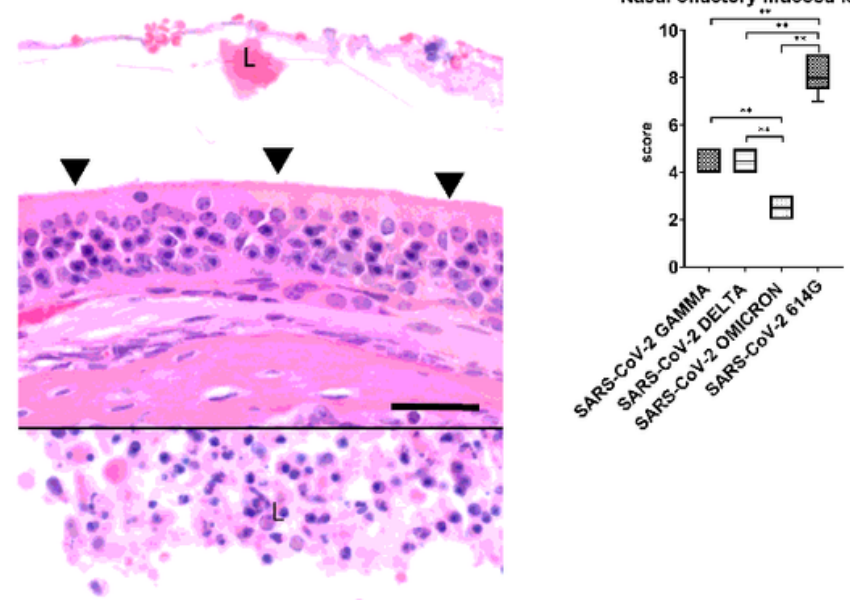

\section{Figure 2}

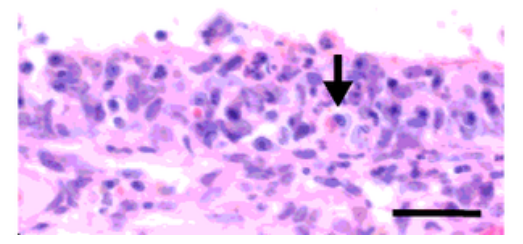

\section{Decreased pathogenicity of VOC Omicron for nasal olfactory mucosa compared to other SARS-CoV-2 strains.}

Olfactory mucosa in SARS-CoV-2 614G or VOCs Gamma and Delta infected hamsters showed the most striking histopathological changes (A, overview). Lesions were mainly characterized by necrosis, single cell apoptosis (A, arrow) and sloughing, associated with intraluminal exudate composed of proteinaceous fluid, cell debris and degenerated neutrophils/heterophils. Olfactory mucosa in Omicron 
infected hamsters was overall intact $(\mathbf{A}$, arrowhead) and with minimal lesions. Semiquantitative analysis of nasal turbinates histopathology (C) revealed significantly lower scores in the overall nasal turbinates, respiratory and olfactory mucosa in the VOC Omicron infected hamsters. Representative images were taken at 20x (scale bar $=500 \mu \mathrm{m}$ ) and 200x (scale bar= $50 \mu \mathrm{m})$ magnification. Data are shown as box and whisker plots with median and quartiles. Significant differences between the infection groups obtained by Mann-Whitney-U tests are indicated by $*\left(* \leq 0.05,{ }^{*} p \leq 0.01\right)$. L=lumen.

A
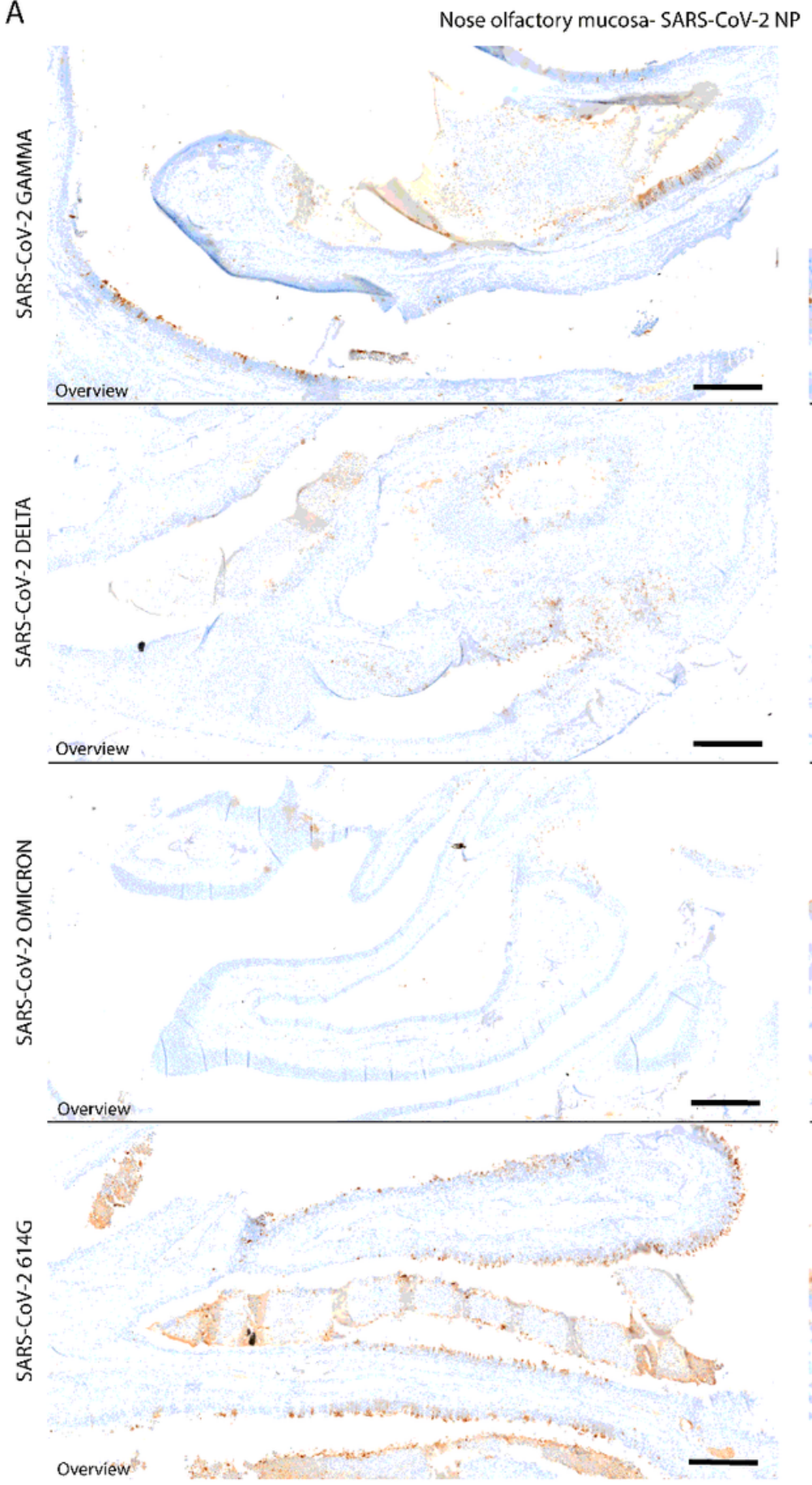
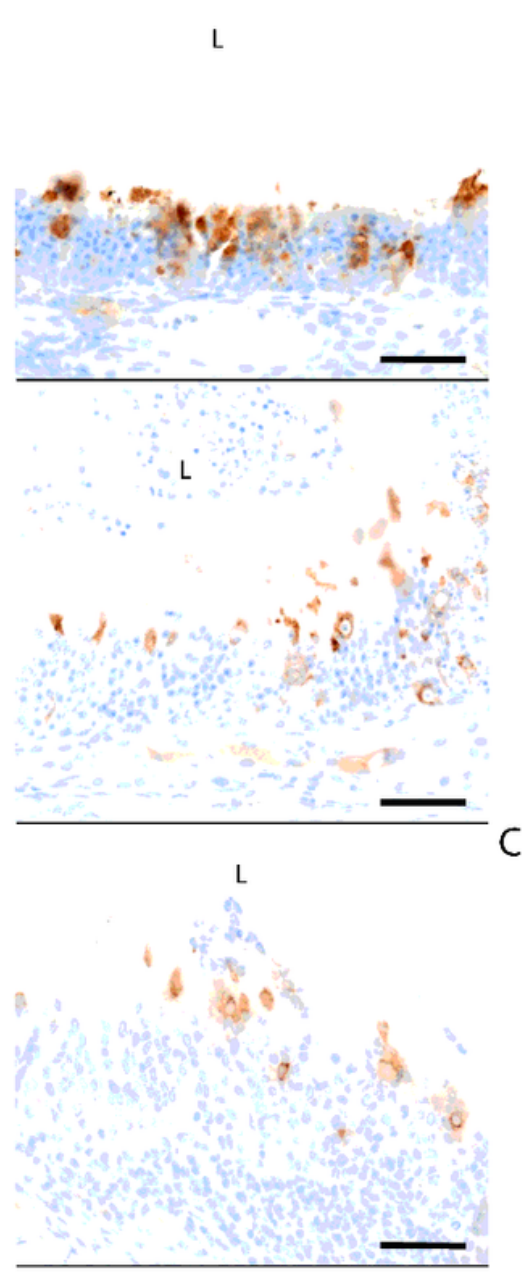

L

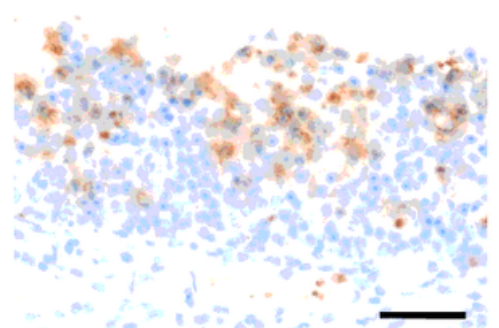

B
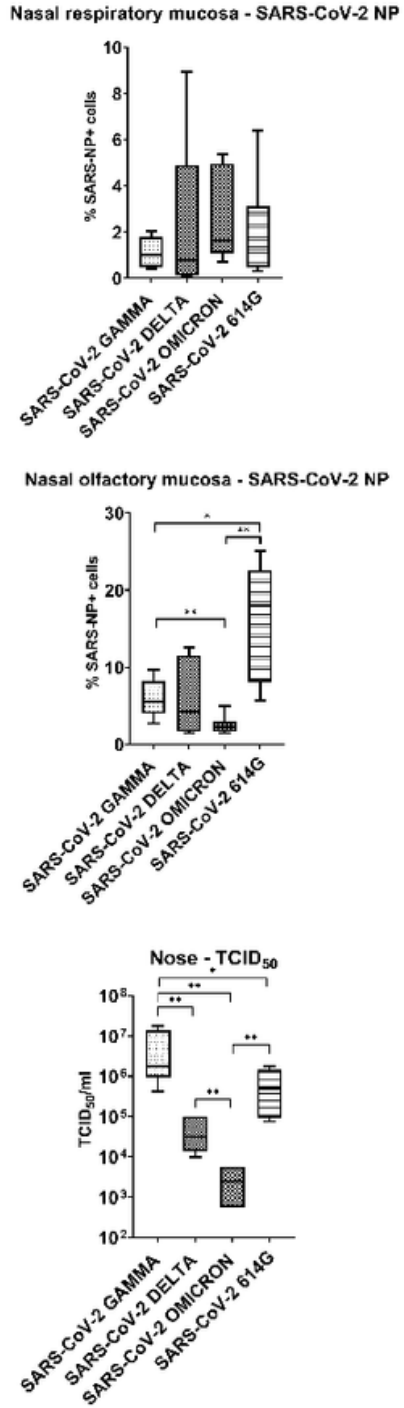

\section{Figure 3}




\section{VOC Omicron infected hamsters showed decreased viral antigen and infectious viral titers in the nasal turbinates compared to other SARS-CoV-2 strains.}

SARS-CoV-2 nucleoprotein (SARS-CoV-2 NP) antigen was mainly detected in the nasal olfactory mucosa (A, overview) of hamsters infected with SARS-CoV-2 614G and VOCs Gamma, Delta. (A,B) Quantification of SARS-CoV-2 NP antigen revealed no significant differences among the groups in the respiratory mucosa of the nose (C). However in the olfactory mucosa VOC Omicron infected hamsters displayed a significantly lower number of SARS-CoV-2+ cells compared to SARS-CoV-2 614G and VOC (C). Infectious SARS-CoV-2 titers, isolated from the nasal turbinates were significantly in the lowest amount in VOC Omicron infected hamsters (D). Representative images of infected Olfactory nasal mucosa were taken at 20x (scale bar $=500 \mu \mathrm{m}$ ) and 200x (scale bar= $50 \mu \mathrm{m}$ ) magnification. Data are shown as box and whisker plots with median and quartiles. Significant differences between the infection groups obtained by MannWhitney $U$ tests are indicated by $*(* \leq 0.05, * * p \leq 0.01)$. L=lumen. 

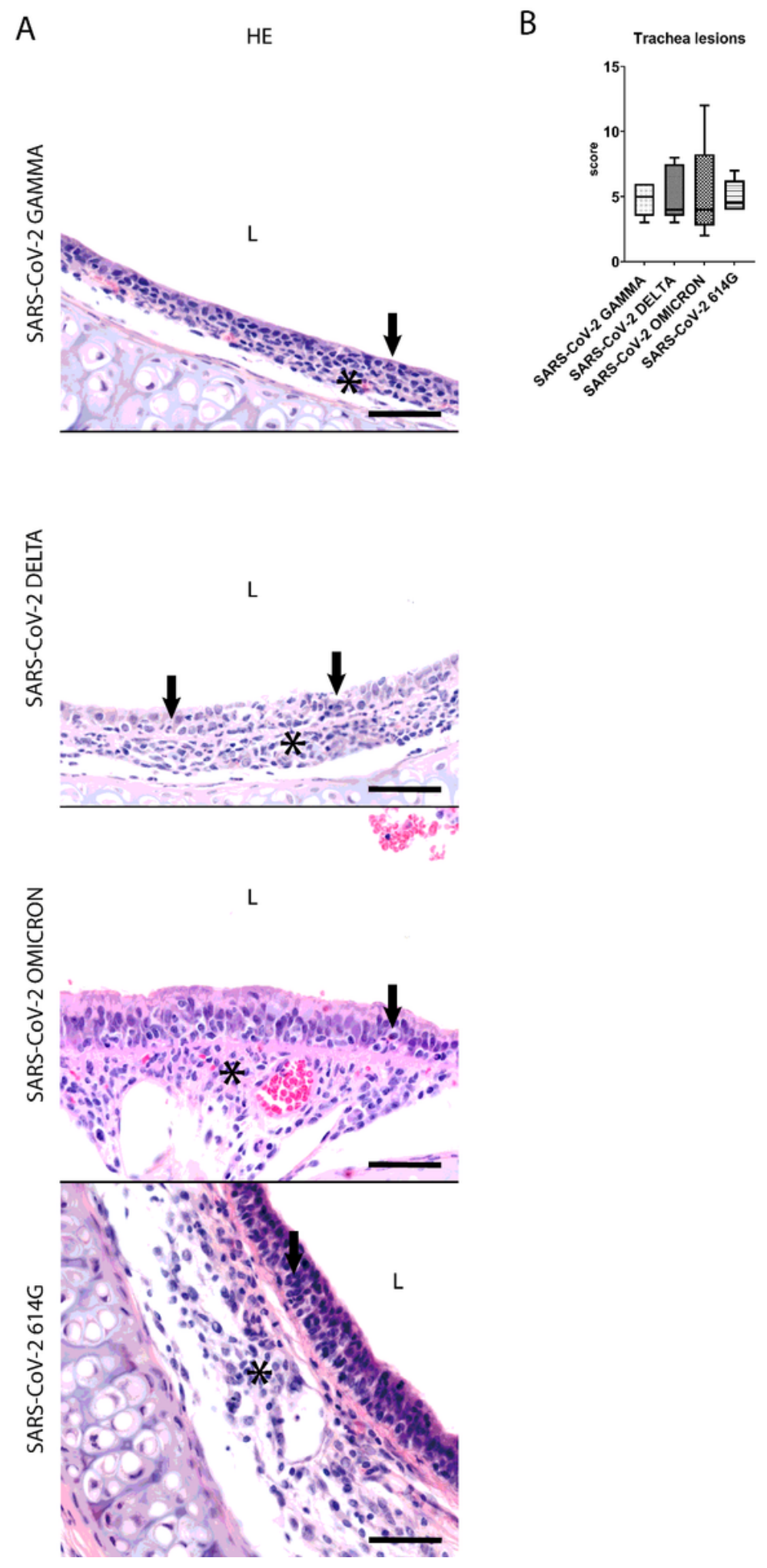

Figure 4

Tracheal lesions are mild to moderate regardless of the SARS-CoV-2 strain infection in hamsters.

Trachea histopathological changes were characterized by neutrophilic/heterophilic exocytosis (arrow) and sub-epithelial infiltration of macrophages and neutrophils/heterophils (asterisks). In addition, scattered ciliary loss was also observed (A). Semiquantitative analysis of tracheal histopathology (B) 
revealed no statistically significant changes in the recorded scores among the groups. Representative images were taken at 200x magnification, scale bar $=50 \mu \mathrm{m}$. Data are shown as box and whisker plots with median and quartiles. L=lumen.
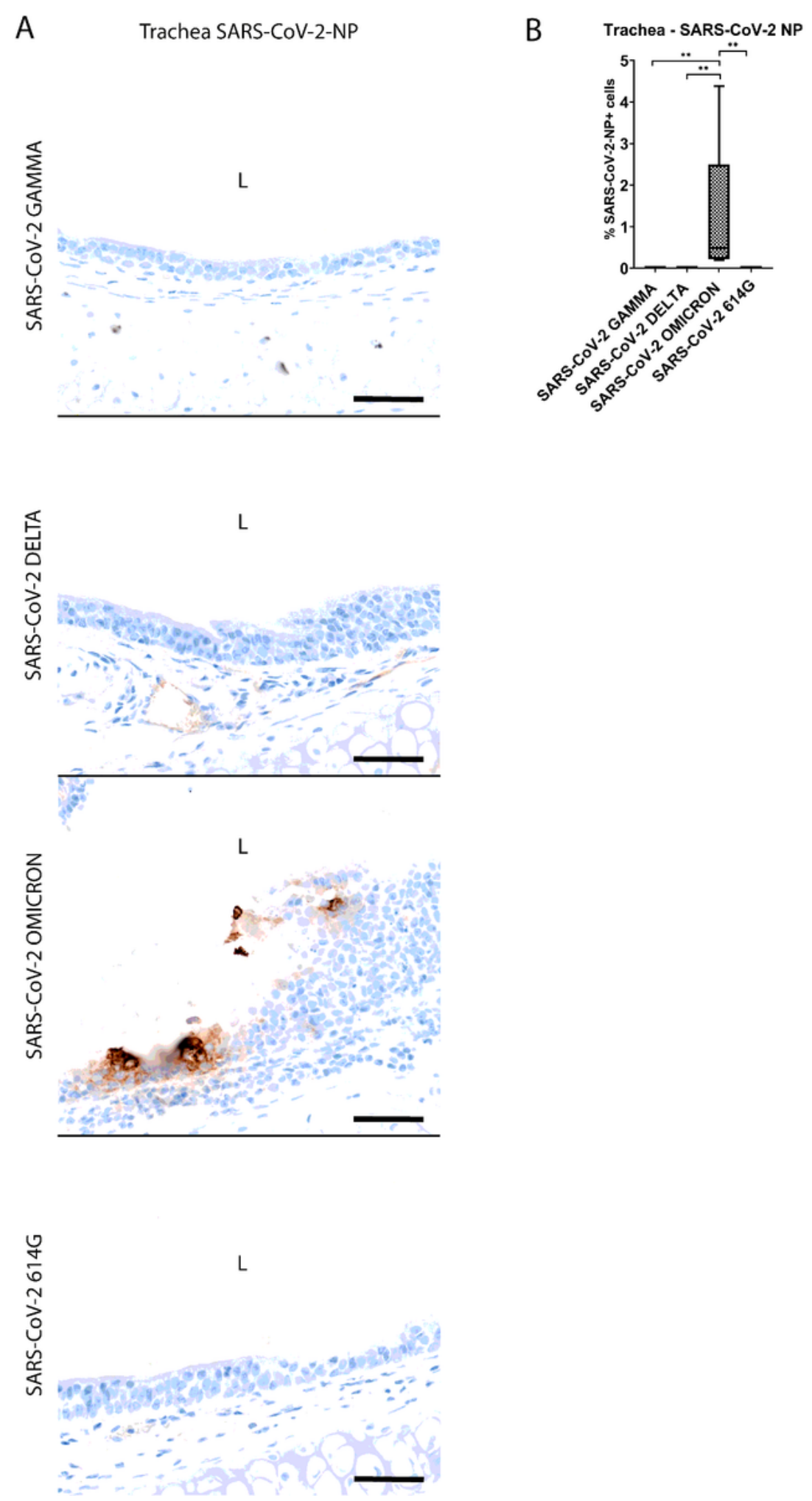

Figure 5 

hamsters.

SARS-CoV-2 nucleoprotein (SARS-CoV-2 NP) antigen was detected in the tracheal epithelium of hamsters infected with VOC Omicron (A). Quantification of SARS-CoV-2 NP antigen revealed a significant higher number of SARS-CoV-2 NP immunolabelled cells in the trachea of VOC Omicron infected hamsters compared to VOCs Gamma and Delta and SARS-CoV-2 614G (B). Representative images of infected nasal mucosa tissue were taken at 200x magnification, Scale bar $=50 \mu \mathrm{m}$. Data are shown as box and whisker plots with median and quartiles. Significant differences between the infection groups obtained by Mann-Whitney $U$ tests are indicated by * $\left.* p \leq 0.05,{ }^{\star *} p \leq 0.01,{ }^{\star \star *} p \leq 0.001\right)$. L=lumen. 
A

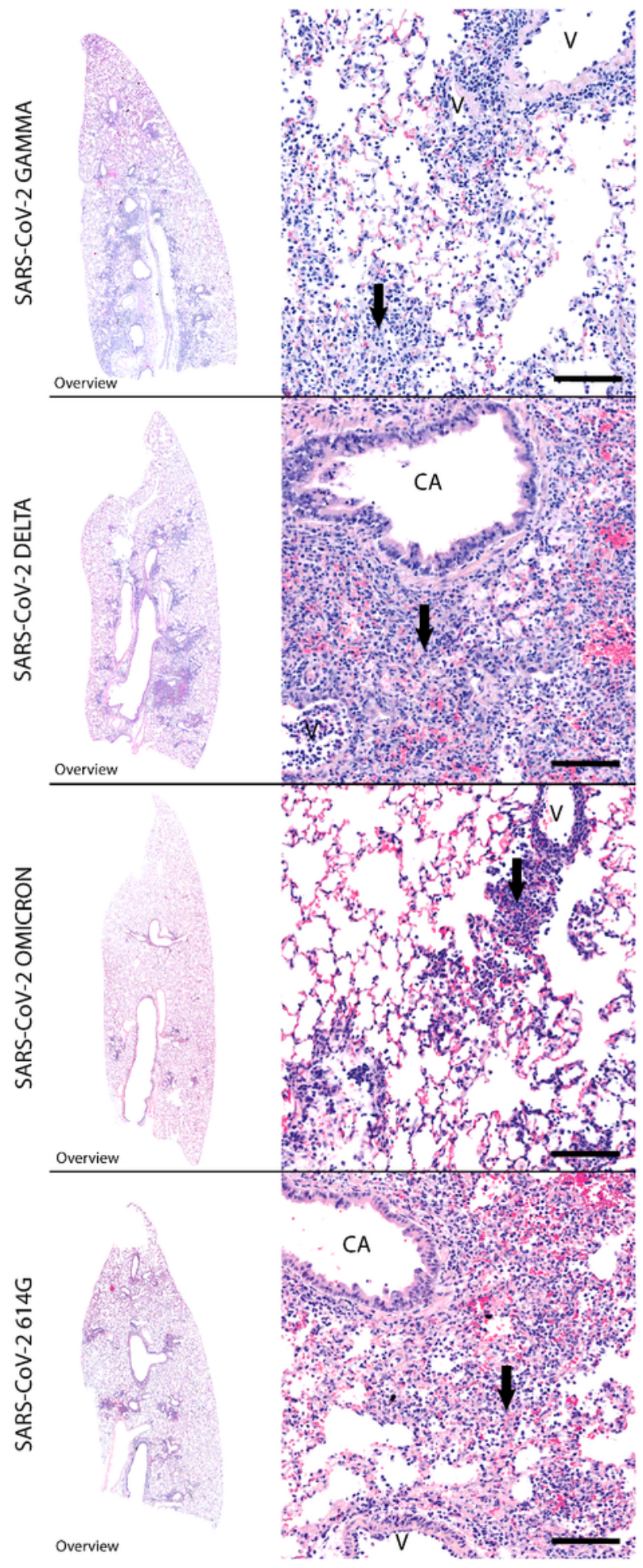

B
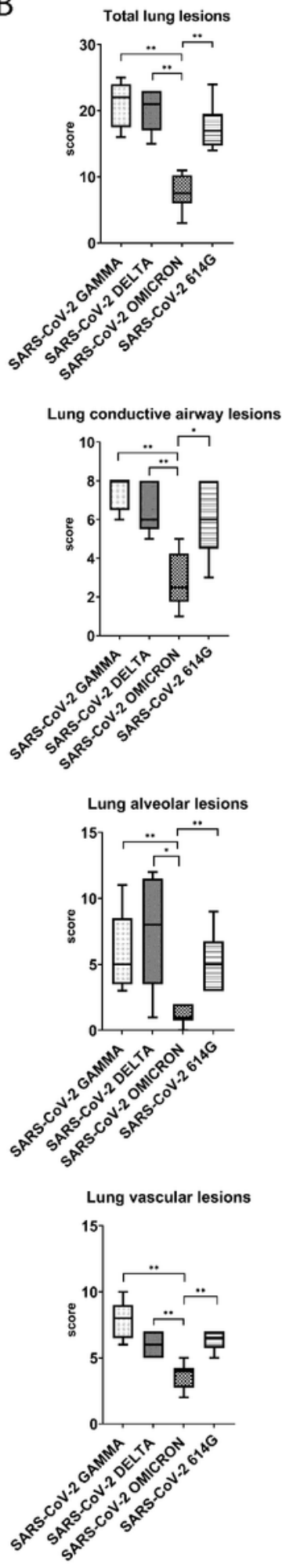

Figure 6

Decreased pathogenicity of VOC Omicron for lungs compared to other SARS-CoV-2 strains.

Lungs of SARS-CoV-2 614 G or VOCs Gamma and Delta infected hamsters showed the most prominent histopathological changes (A, overview) Lesions were characterized by alveolar septal infiltration and obscuration by macrophages and neutrophils/heterophils admixed with extravasated erythrocytes and 
fibrin (A, arrow). Conductive airways frequently showed epithelial hyperplasia (A, CV). Vascular lesions mainly consisted of histiocytic-neutrophilic/heterophilic perivascular cuff and intramural infiltrates (A, V). Lungs of Omicron infected animals were mildly affected with a marked affected and not in all lung compartmens. Semiquantitative analysis of pulmonary histopathology (B) revealed significantly lower scores in the overall lung, lung conductive airways, lung alveoli, and lung vascular compartment in the VOC Omicron infected hamsters compared to the other investigated groups. Representative images were taken at 20x and 100x magnification, scale bar $=50 \mu \mathrm{m}$. Data are shown as box and whisker plots with median and quartiles. Significant differences between the infection groups obtained by Mann-Whitney-U tests are indicated by * $(* p \leq 0.05, * \star p \leq 0.01)$. 
A
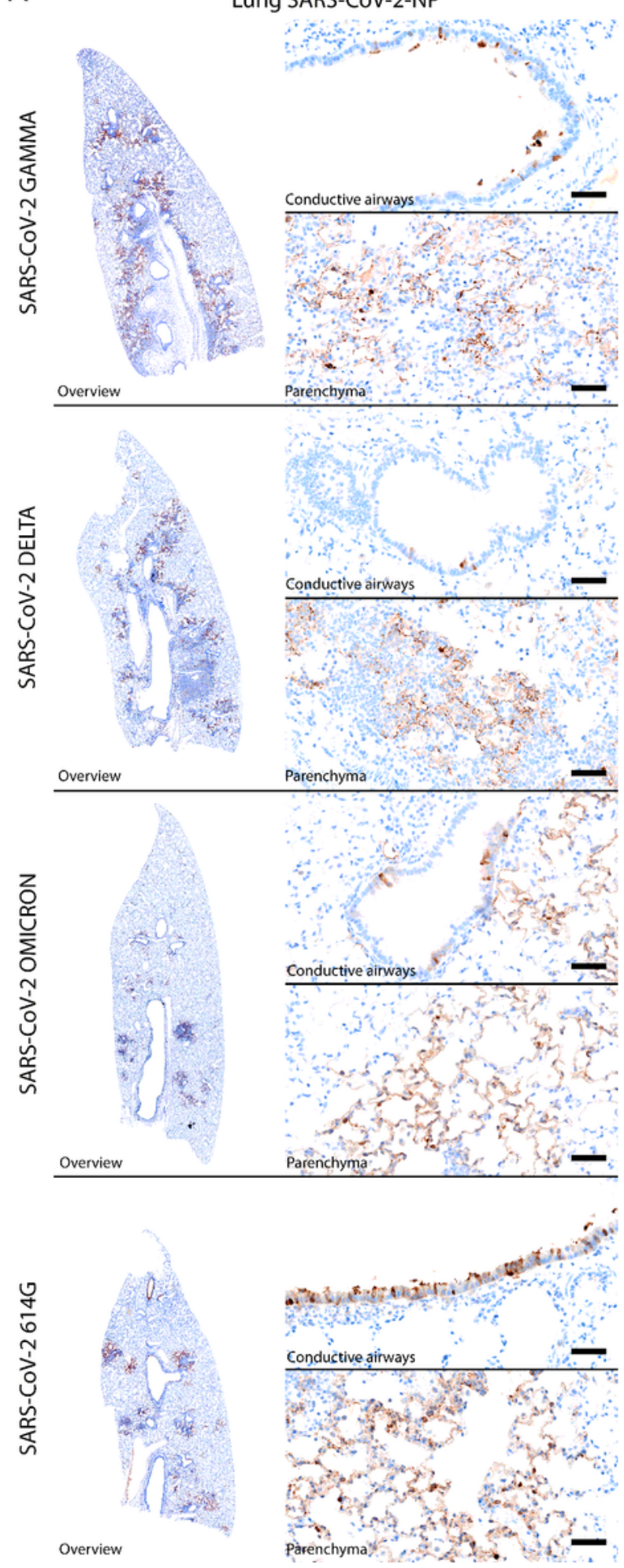

B

Conductive airways - SARS-CoV-2 NP

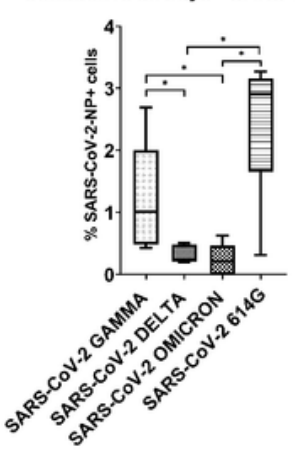

Parenchyma - SARS-CoV-2 NP

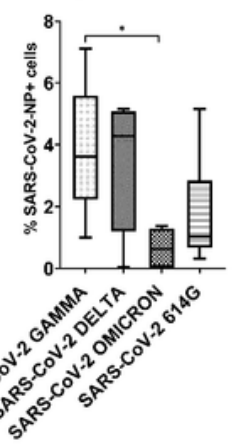

C

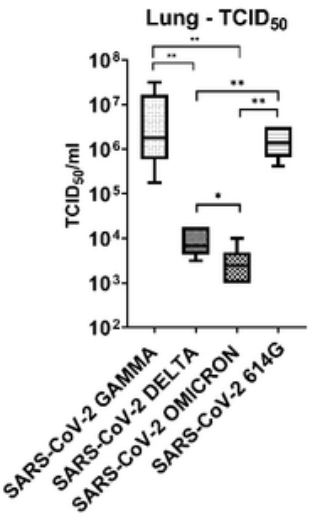

\section{Figure 7}

VOC Omicron infected hamsters showed decreased viral antigen and infectious viral titers in the lung compared to other SARS-CoV-2 strains.

All SARS-CoV-2 infected hamsters showed SARS-CoV-2 nucleoprotein (SARS-CoV-2 NP) immunolabelled cells, but the distribution and the amount varied among the groups (A, Overview). SARS-CoV-2 NP 
immunolabelled cells were detected in the lung conductive airways and in the lung parenchyma in variable amount according to the investigated group (A). Quantification of SARS-CoV-2 NP antigen revealed significantly lower numbers of SARS-CoV-2 NP immunolabelled cells in the lung conductive airways of VOC Omicron infected hamsters compared to VOC Gamma and SARS-CoV-2 614G infected ones (B). VOC Omicron infected hamsters showed also a significantly lower numbers of SARS-CoV-2 NP immunolabelled cells in the lung parenchyma compared to VOC Gamma (B). Infectious SARS-CoV-2 titers, isolated from the lung were significantly in the lowest amount in VOC Omicron infected hamsters (C). Representative images were taken at 200x magnification, scale bar= $50 \mu \mathrm{m}$. Data are shown as box and whisker plots with median and quartiles. Significant differences between the infection groups obtained by Mann-Whitney-U tests are indicated by * ${ }^{*} p \leq 0.05$, $\left.{ }^{*} p \leq 0.01\right)$.

\section{Supplementary Files}

This is a list of supplementary files associated with this preprint. Click to download.

- SupplementaryTables.docx 